\title{
TOP PAIR PRODUCTION CROSS-SECTION MEASUREMENT IN THE ALL-HADRONIC CHANNEL AT CDF AND D
}

\author{
DANIEL WICKE \\ Fermilab, P.O. Box 500 \\ Batavia, Illinois 60510, USA*
}

on behalf of the CDF and the DØ Collaborations

\begin{abstract}
Measurements of the $t \bar{t}$ production cross-section at $\sqrt{s}=1.96 \mathrm{TeV}$ in proton-antiproton collisions were performed by the CDF and $\mathrm{D} \varnothing$ collaborations using $t \bar{t}$ final states where both $W$ 's decay hadronically. Each experiment uses luminosity of about $160 \mathrm{pb}^{-1}$ of data collected during Run II of the Fermilab Tevatron collider. Beside kinematical observables both experiments take advantage of identifying $b$ jets based on lifetime tagging. While CDF counts the number of $b$-tags over background after a cut based analysis, D $\varnothing$ counts the excess of events after a neural network based kinematical selection and requiring a $b$-tagged jet.

CDF obtains $\sigma_{t \bar{t}}=7.8 \pm 2.5_{\text {stat }}{ }_{-2.3}^{+4.7}$ syst $; \quad \mathrm{D} \varnothing \sigma_{t \bar{t}}=7.7_{-3.3}^{+3.4}$ stat ${ }_{-3.8}^{+4.7}$ syst $\pm 0.5_{\text {lumi }}$.
\end{abstract}

Keywords: top; cross-section; Tevatron.

\section{Introduction}

The measurement of the $t \bar{t}$ cross-section is an important cross check of our understanding of the top-production mechanism and of the completeness of its decay modes. Such measurements are performed in the "dilepton", the "lepton+jets" and the "all hadronic" decay channel, which are named after the decays of the $W$ 's, because in the Standard Model the top almost always decays to a $b$ and a $W$.

This note summarises the measurements done in the "all hadronic" channel by $\mathrm{CDF}^{1}$ and $\mathrm{D} Q^{2}$ in $p \bar{p}$-collisions at $\sqrt{s}=1.96 \mathrm{TeV}$. This channel has the highest branching ratio of $44 \%$, however, it suffers from an overwhelming background from light quark pair-production which has many orders of magnitude higher crosssection. The additional jets faking the hadronically decaying $W$ 's stem from hard gluon radiation and splitting.

Following the pieces of the master formula for cross-section measurements

$$
\sigma_{t \bar{t}}=\frac{N-B}{\varepsilon \mathcal{L} \cdot \mathrm{BR}}
$$

${ }^{*}$ on leave of absence from Bergische Universität Wuppertal 
first the selection of signal events in this environment, then the background and the efficiency determination are discussed. The branching ratio, BR, is taken from standard model predictions 3445 . Luminosity measurements for CDF and D $\varnothing$ are discussed elsewhere 6 67.

\section{Signal Selection}

\subsection{Trigger and Preselection}

At $p \bar{p}$ colliders signal selection begins with the trigger. To select all hadronic $t \bar{t}$ events $\mathrm{CDF}$ and $\mathrm{D} \varnothing$ require 4 clusters with a transverse energy above $15 \mathrm{GeV}$ and $12 \mathrm{GeV}$, respectively. CDF in addition requires a minimal value for the scalar sum of all transverse momenta $H_{T} \geq 125 \mathrm{GeV}$, while $\mathrm{D} \varnothing$ asks the two hardest jets to have $p_{T}>25 \mathrm{GeV}$ and the third jet $p_{T}>15 \mathrm{GeV}$.

Both experiments confirm their trigger selection with offline objects and veto on isolated high $p_{T}$ leptons to keep the analyses orthogonal to their measurements in the other channels. While D $\varnothing$ continues with 6 or more jet events CDF keeps the 4 jet events for calibration in the background determination.

Both experiments work with an integrated luminosity of around $160 \mathrm{pb}^{-1}$.

\subsection{Final Signal Selection}

General kinematic observables can distinguish between $t \bar{t}$-signal and multi-jet background. Top events are expected on average to have a larger overall energy scale and harder sub-leading jets. Their shape is expected to be more spherical and less planar. Their rapidity distribution is more central than that of light quark production.

\subsubsection{Kinematic Observables}

To select signal events CDF uses the sum of all transverse momenta, $H_{T}$, the sum of the transverse momenta without the two leading jets, $H_{T}^{3 \mathrm{j}}$, Aplanarity and Centrality for the signal selection.

$\mathrm{D} \varnothing$ uses in addition $\sqrt{s}$, the geometric mean of the 5 th and 6 th jets $E_{T}, E_{T_{5,6}}$, the $E_{T}$ weighted number of jets, $N_{\text {jets }}^{A}$, Sphericity and a momentum weighted rapidity moment, $\left\langle\eta^{2}\right\rangle$. D $\varnothing$ also exploits observables obtained from assuming a $t \bar{t}$-event and reconstructing the decay chain. The consistency of the reconstructed $W$ - and $t$ masses with the known values, the invariant $t \bar{t}$-mass, the invariant $W W$-mass as well as the highest and the second highest dijet-mass are considered.

\subsubsection{B-Tagging}

Besides the kinematic properties the presence of $b$-jets in $t \bar{t}$-events is an important feature that is used in the signal selection. Both CDF and $D \varnothing$ are equipped with silicon vertex detectors which are used to reconstruct displaced secondary vertices stemming from $B$-decays. Such secondary vertices are reconstructed using tracks 
belonging to a jet of interest. The significance of the displacement of the secondary vertex from the primary vertex is used to decide whether the jet originates from a $b$-quark. CDF requires a significance of $3, \mathrm{D} \varnothing$ one of 7 to call a jets $b$-tagged.

\subsubsection{Cut Procedures}

To have optimal cuts in the presence of correlated observables D $\varnothing$ uses a series of neural networks $(\mathrm{NN})$ for the selection. A first neural net (NN0) uses a subset of kinematic information to perform a final step of loose preselection. Then one $b$-tag is required. The next NN combines all kinematic observables into a single number which is then fed into the final neural net (NN2) which includes the reconstructed top-properties. After cutting on the output of NN2 DØ selects 220 events.

CDF uses straight cuts in which a linear combination of Aplanarity and $H_{T}^{3 \mathrm{j}}$ is used to find an optimal cut for these correlated observables. After the kinematic cuts CDF counts 326 b-tags.

\section{Background Estimation}

Both experiments estimate their background contribution directly from data by removing the actual $b$-tag and instead weighting the events with the probability of a random event to be $b$-tagged, the so called Tag Rate Function (TRF). To provide a background estimate, the TRFs must be measured on background samples.

CDF uses the 4-jet events after the preselection to measure their TRF as function of $p_{T}, \eta$, number of tracks at the primary vertex, total number of tracks and Aplanarity. D $\varnothing$ uses all preselected events (after NN0) and parametrises the TRF as function of $p_{T}$ and $\eta$ in 4 bins of $H_{T}$.

The validity of the TRFs is checked by comparing distributions obtained by applying the TRF on the data with those obtained using the actual $b$-tag.

From these studies CDF expects $264.7 \pm 17.2$ background tags thus claiming 61 surplus $b$-tags. D $\varnothing$ expects $186 \pm 14.8$ background events, i.e. sees an excess of 34 events. The uncertainties are obtained by repeating the analysis with TRFs obtained from samples with varied selection cuts.

\section{Efficiency Determination}

The efficiency for the $t \bar{t}$ signal to survive the applied cuts is obtained from $t \bar{t}$ simulation. The obtained total efficiencies are $0.047 \pm 0.010$ and $0.058 \pm 0.001_{\text {stat }} \pm 0.018_{\text {syst }}$ for CDF and DØ, respectively. The CDF efficiency includes a factor of two needed to account for the expected two $b$-tags in an ideal experiment when applying Eq. (11). CDF also neglected their trigger efficiency, which is believed to be above $95 \%$.

The two experiments investigated several systematic uncertainties. In both cases the by far dominating systematic uncertainty comes from the jet energy scale resolution. It accounts for $28 \%$ uncertainty on the efficiency. Other uncertainties investigated are below $10 \%$. 


\section{Summary and Results}

CDF and D $\varnothing$ preformed a measurement of the $t \bar{t}$ cross-section in the all-hadronic channel. Combining the described background estimates from data and efficiencies obtained from simulation with the luminosity of each experiment the following results are obtained:

$$
\mathrm{CDF}: \sigma_{t \bar{t}}=7.8 \pm 2.5_{\text {stat }}{ }_{-2.3 \text { syst }}^{+4.7} \quad \mathrm{D} \varnothing: \sigma_{t \bar{t}}=7.7_{-3.3}^{+3.4} \text { stat }{ }_{-3.8}^{+4.7} \text { syst } \pm 0.5_{\text {lumi }}
$$

The dominating systematic uncertainties in the final result are the jet energy scale uncertainty of the selection efficiency and the uncertainty of the TRF-based background prediction, which gets inflated due to the subtraction in Eq. (11).

\section{References}

1. The CDF Collaboration. Measurement of the $t \bar{t}$ production cross section in the all-hadronic channel. CDF note 7075, August 2004.

2. The $D \varnothing$ Collaboration. Measurement of the $t \bar{t}$ cross section in the all-jets channel. D $\varnothing$ note 4428, April 2004.

3. Edmond L. Berger and Harry Contopanagos. Threshold resummation of the total cross section for heavy quark production in hadronic collisions. Phys. Rev. D57(1998) 253-264.

4. Roberto Bonciani et al. NLL resummation of the heavy-quark hadroproduction crosssection. Nucl. Phys. B529(1998) 424-450.

5. Nikolaos Kidonakis and Ramona Vogt. Next-to-next-to-leading order soft-gluon corrections in top quark hadroproduction. Phys. Rev. D68(2003) 114014.

6. CDF Collaboration, D. Acosta et al. The CDF Cherenkov luminosity monitor. Nucl. Instrum. Meth. A461(2001) 540-544.

7. T. Edwards et al. Determination of the effective inelastic $p \bar{p}$ cross-section for the D $\varnothing$ Run II luminosity measurement. FERMILAB-TM-2278-E, 2004.
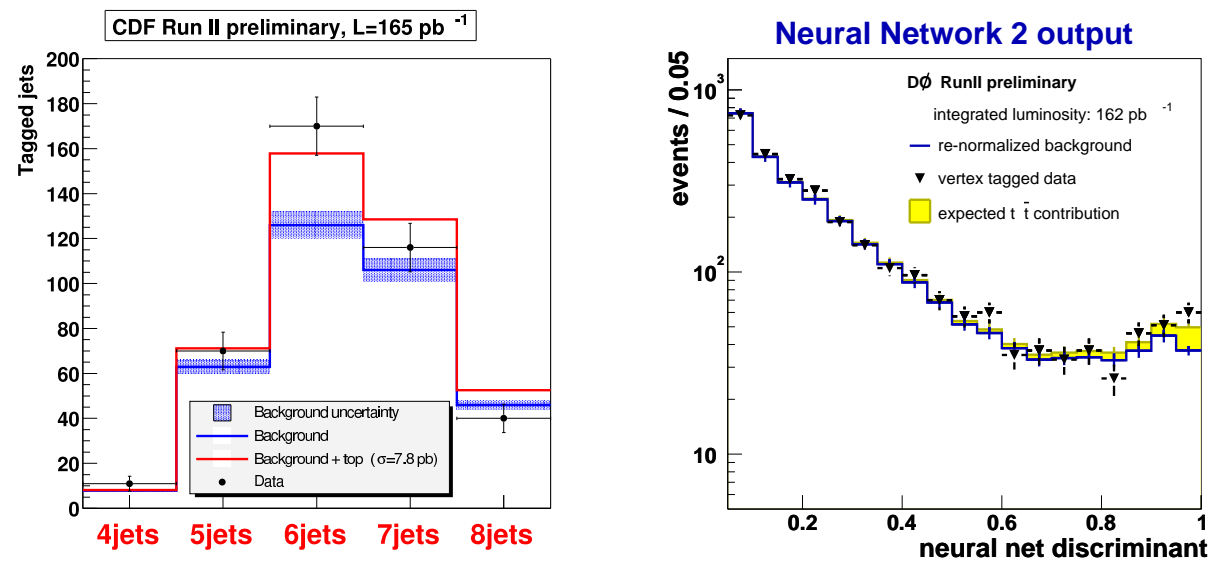

Fig. 1. CDF (left): Number of $b$-tags vs. number of jets per event. Signal is at 6 and above. $\mathrm{D} \varnothing$ (right): The final neural network (NN2) output (required to be above 0.75 for signal). Background estimates and a Standard Model expectation are shown by both experiments. 